Journal of Applied AnALysis

Vol. 13, No. 1 (2007), pp. 57-76

\title{
ON MULTIVALUED COSINE FAMILIES
}

\author{
M. PISZCZEK
}

Received July 27, 2004 and, in revised form, December 28, 2005

\begin{abstract}
Let $K$ be a convex cone in a real Banach space. The main purpose of this paper is to show that for a regular cosine family $\left\{F_{t}\right.$ : $t \in \mathbb{R}\}$ of linear continuous multifunctions $F_{t}: K \rightarrow c c(X)$ there exists a linear continuous multifunction $H: K \rightarrow c c(K)$ such that

$$
F_{t}(x) \subset \sum_{n=0}^{\infty} \frac{t^{2 n}}{(2 n) !} H^{n}(x) .
$$
\end{abstract}

Let $X$ be a real normed vector space. We will denote by $n(X)$ the family of all nonempty subsets of $X$ and by $c c(X)$ the family of all nonempty compact and convex subsets of $X$.

For $A, B \subset X$ and $t \in \mathbb{R}$ we introduce

$$
A+B=\{a+b: a \in A, b \in B\}, \quad t A=\{t a: a \in A\} .
$$

A subset $K$ of $X$ is called a cone if $t K \subset K$ for all $t \in(0,+\infty)$. A cone is said to be convex if it is a convex set.

Let $A, B, C$ be sets of $c c(X)$. We say that the set $C$ is the Hukuhara difference of $A$ and $B$, i.e., $C=A-B$ if $B+C=A$. By the Rådström Lemma [12] it follows that if this difference exists, then it is unique.

2000 Mathematics Subject Classification. Primary: 26E25, 28B20, 47D09.

Key words and phrases. Riemann integral of set-valued functions, multivalued cosine families.

ISSN 1425-6908（C) Heldermann Verlag. 
A multifunction $F:[a, b] \rightarrow c c(X)$ is called concave if

$$
F(\lambda t+(1-\lambda) s) \subset \lambda F(t)+(1-\lambda) F(s)
$$

for all $s, t \in[a, b]$ and $\lambda \in(0,1)$.

We say that a multifunction $F: J \rightarrow c c(X)$, where $J$ denotes an interval in $\mathbb{R}$, is increasing if for all $s, t \in J$ such that $s<t$ we have $F(s) \subset F(t)$. If for $s<t$ we have the inverse inclusion $F(t) \subset F(s)$, then the multifunction is called decreasing.

We call $F: \mathbb{R} \rightarrow c c(X)$ even if $F(-t)=F(t)$ for every $t \in \mathbb{R}$.

Let $K$ be a convex cone in $X$. A multifunction $F: K \rightarrow n(K)$ is called linear if

$$
F(x+y)=F(x)+F(y), \quad F(\lambda x)=\lambda F(x)
$$

for all $x, y \in K$ and $\lambda \geq 0$.

The image of a set $A \subset K$ by $F: K \rightarrow n(X)$ is the set

$$
F(A)=\bigcup_{y \in A} F(y)
$$

Let $X, Y, Z$ be nonempty sets. The superposition $G \circ F$ of multifunctions $F: X \rightarrow n(Y)$ and $G: Y \rightarrow n(Z)$ we define by the formula

$$
(G \circ F)(x)=G(F(x)) \quad \text { for } x \in X .
$$

Let $A, A_{1}, A_{2}, \ldots$ be elements of the family $c c(X)$. We say that the sequence $\left(A_{n}\right)_{n \in \mathbb{N}}$ is convergent to $A$ and we write $A_{n} \rightarrow A$ if $d\left(A, A_{n}\right) \rightarrow 0$, where $d$ denotes the Hausdorff metric derived by the norm in $X$.

Lemma 1 ([11, Lemma 1]). Let $X$ be a real Banach space, $A, A_{1}, A_{2}, \ldots$, $B, B_{1}, B_{2}, \ldots \in c c(X)$. If $A_{n} \rightarrow A, B_{n} \rightarrow B$ and there exist the Hukuhara differences $A_{n}-B_{n}$ in $c c(X)$ for $n \in \mathbb{N}$, then there exists the Hukuhara difference $A-B$ and $A_{n}-B_{n} \rightarrow A-B$.

The norm $\|A\|$ of a bounded set $A \subset X$ is defined by

$$
\|A\|:=\sup \{\|a\|: a \in A\}=d(A,\{0\}) .
$$

Next we introduce the Hukuhara version of the Riemann integral of multifunction $F:[a, b] \rightarrow c c(X)$ (see [2]). We will denote by $\Delta=\left\{\alpha_{0}, \alpha_{1}, \ldots, \alpha_{n}\right\}$ a partition of the interval $[a, b]$, i.e., a sequence satisfying inequalities $a=\alpha_{0}<\alpha_{1}<\ldots<\alpha_{n}=b$. The number

$$
\delta(\Delta)=\max \left\{\alpha_{i+1}-\alpha_{i}: i=0,1, \ldots, n-1\right\}
$$

is said to be the diameter of $\Delta$. $\Phi$ denotes the family of all pairs $(\Delta, \tau)$, where $\Delta=\left\{\alpha_{0}, \alpha_{1}, \ldots, \alpha_{n}\right\}$ is a partition of the interval $[a, b]$ and $\tau=$ 
$\left(\tau_{0}, \ldots, \tau_{n-1}\right)$ is a sequence of points such that $\tau_{i} \in\left[\alpha_{i}, \alpha_{i+1}\right] . \quad I(\Delta, \tau)$ denotes the set

$$
I(\Delta, \tau)=\sum_{i=0}^{n-1}\left(\alpha_{i+1}-\alpha_{i}\right) F\left(\tau_{i}\right)
$$

for $(\Delta, \tau) \in \Phi$. If $I(\Delta, \tau)$ converges to $I \in c c(X)$ with respect to the Hausdorff metric $d$ when $\delta(\Delta) \rightarrow 0$, i.e.,

$$
(\forall \varepsilon>0)(\exists \eta>0)(\forall(\Delta, \tau) \in \Phi)(\delta(\Delta)<\eta \Longrightarrow d(I(\Delta, \tau), I)<\varepsilon),
$$

then we say that $I$ is the integral of the multifunction $F$ on the interval $[a, b]$ and we write

$$
I=\int_{a}^{b} F(t) d t
$$

If there exists the integral of a multifunction $F:[a, b] \rightarrow c c(X)$, then we say that $F$ is integrable.

Next lemmas describe some properties of the Riemann integral for multifunctions.

Lemma 2 ([2, p. 212]). If $a<c<b$ and $F:[a, b] \rightarrow c c(X)$ is integrable on $[a, c]$ and on $[c, b]$, then $F$ is integrable on $[a, b]$ and

$$
\int_{a}^{b} F(t) d t=\int_{a}^{c} F(t) d t+\int_{c}^{b} F(t) d t .
$$

Lemma 3 ([2, p. 212]). Let $X$ be a real Banach space. If $F:[a, b] \rightarrow c c(X)$ is integrable on $[a, b]$, then for every $c \in(a, b) F$ is integrable on $[a, c]$ and on $[c, b]$ and formula (1) holds.

Lemma 4 ([10, Lemma 1.3]). If $F:[a, b] \rightarrow c c(X)$ is integrable, $a^{\prime}, b^{\prime}, A$, $B$ are real numbers such that $a^{\prime}<b^{\prime}, A a^{\prime}+B=a, A b^{\prime}+B=b$, then

$$
\int_{a}^{b} F(t) d t=A \int_{a^{\prime}}^{b^{\prime}} F(A u+B) d u .
$$

Lemma 5 ([10, Lemma 1.4]). Let $F, G:[a, b] \rightarrow c c(X)$ be integrable. If $F(t) \subset G(t)$ for all $t \in[a, b]$, then

$$
\int_{a}^{b} F(t) d t \subset \int_{a}^{b} G(t) d t .
$$


Lemma 6. If $F: \mathbb{R} \rightarrow c c(X)$ is integrable on each interval $[a, b]$ and even, then

$$
\int_{a}^{b} F(t) d t=\int_{-b}^{-a} F(t) d t
$$

Proof. Let $\Delta=\left\{\alpha_{0}, \alpha_{1}, \ldots, \alpha_{n}\right\}$ be a partition of the interval $[a, b]$ and $\tau_{i} \in\left[\alpha_{i}, \alpha_{i+1}\right]$ for $i \in\{0,1, \ldots, n-1\}$. Then $\bar{\Delta}=\left\{\beta_{0}, \beta_{1}, \ldots, \beta_{n}\right\}$, where $\beta_{i}=-\alpha_{n-i}$ is partition of $[-b,-a]$. By the evenness of the multifunction $F$ we have the equality of integral sums

$$
I(\Delta, \tau)=\sum_{i=0}^{n-1}\left(\alpha_{i+1}-\alpha_{i}\right) F\left(\tau_{i}\right)=\sum_{i=0}^{n-1}\left(-\alpha_{i}-\left(-\alpha_{i+1}\right)\right) F\left(-\tau_{i}\right)=I(\bar{\Delta}, \bar{\tau}),
$$

where $\overline{\tau_{i}}=-\tau_{n-1-i}, \bar{\tau}=\left(\overline{\tau_{0}}, \overline{\tau_{1}}, \ldots, \overline{\tau_{n-1}}\right)$. Let $\left(\Delta_{p}\right)$ be a normal sequence of partitions of the interval $[a, b]$ and $\left(\overline{\Delta_{p}}\right)$ be corresponding normal sequence of partitions of the interval $[-b,-a]$. Then $I\left(\Delta_{p}, \tau\right)=I\left(\overline{\Delta_{p}}, \bar{\tau}\right)$, hence and by integrability of $F$ we have (2).

Lemma 7. Let $X$ be a real Banach space. If $F:[0, \infty) \rightarrow c c(X)$ is integrable on each interval $[a, b] \subset[0, \infty)$ where $0 \leq a \leq b<\infty$ and there exist the Hukuhara differences $F(t)-F(s)$ for $0 \leq s \leq t$, then for $u \geq 0$ there exists the Hukuhara difference

$$
\int_{a+u}^{b+u} F(t) d t-\int_{a}^{b} F(t) d t
$$

Proof. Let $\Delta=\left\{\alpha_{0}, \alpha_{1}, \ldots, \alpha_{n}\right\}$ be a partition of the interval $[a, b]$ and $\tau_{i} \in$ $\left[\alpha_{i}, \alpha_{i+1}\right]$ for $i \in\{0,1, \ldots, n-1\}$. Then $\Delta^{u}=\left\{\alpha_{0}+u, \alpha_{1}+u, \ldots, \alpha_{n}+u\right\}$ is the partition of the interval $[a+u, b+u]$ and $\tau_{i}+u \in\left[\alpha_{i}+u, \alpha_{i+1}+u\right]$. We consider integral sums corresponding to these partitions

$$
I(\Delta, \tau)=\sum_{i=0}^{n-1}\left(\alpha_{i+1}-\alpha_{i}\right) F\left(\tau_{i}\right)
$$

and

$$
I\left(\Delta^{u}, \tau_{u}\right)=\sum_{i=0}^{n-1}\left(\left(\alpha_{i+1}+u\right)-\left(\alpha_{i}+u\right)\right) F\left(\tau_{i}+u\right),
$$

where $\tau_{u}=\left(\tau_{0}+u, \tau_{1}+u, \ldots, \tau_{n-1}+u\right)$. Since for $i \in\{0,1, \ldots, n-1\}$ the Hukuhara differences $F\left(\tau_{i}+u\right)-F\left(\tau_{i}\right)$ exist, it follows that there exists the 
Hukuhara difference

$$
I\left(\Delta^{u}, \tau_{u}\right)-I(\Delta, \tau)=\sum_{i=0}^{n-1}\left(\alpha_{i+1}-\alpha_{i}\right)\left(F\left(\tau_{i}+u\right)-F\left(\tau_{i}\right)\right) .
$$

Let $\left(\Delta_{p}\right)$ be a normal sequence of partitions of the interval $[a, b]$ and $\left(\Delta_{p}^{u}\right)$ be corresponding normal sequence of partitions of the interval $[a+u, b+u]$. Since $F$ is integrable on each interval we have

$$
I\left(\Delta_{p}, \tau\right) \rightarrow \int_{a}^{b} F(t) d t \quad \text { and } \quad I\left(\Delta_{p}^{u}, \tau_{u}\right) \rightarrow \int_{a+u}^{b+u} F(t) d t \quad \text { when } p \rightarrow \infty .
$$

Hence and by Lemma 1 there exists the Hukuhara difference

$$
\int_{a+u}^{b+u} F(t) d t-\int_{a}^{b} F(t) d t
$$

In next lemmas we assume that $X$ is a real Banach space.

Lemma 8 ([2, p. 212]). If $F: \mathbb{R} \rightarrow c c(X)$ is continuous, then it is integrable on each interval $[a, b] \subset \mathbb{R}$.

Lemma 9 ([2, p. 211]). If $F:[a, b] \rightarrow c c(X)$ is continuous, then

$$
\left\|\int_{a}^{b} F(t) d t\right\| \leq \int_{a}^{b}\|F(t)\| d t
$$

Lemma 10. If $F: \mathbb{R} \rightarrow c c(X)$ is continuous, then for every $a \in \mathbb{R}$ the multifunction

$$
H(t)=\int_{a}^{t} F(u) d u \quad \text { for } t \geq a
$$

is continuous.

Proof. Let $h>0$ and $t \geq a$. By Lemmas 3 and 9 and properties of the Hausdorff metric we have

$$
d(H(t), H(t+h))=d\left(\int_{a}^{t} F(u) d u, \int_{a}^{t} F(u) d u+\int_{t}^{t+h} F(u) d u\right)
$$




$$
=\left\|\int_{t}^{t+h} F(u) d u\right\| \leq \int_{t}^{t+h}\|F(u)\| d u \leq h \sup _{t \leq u \leq t+h}\|F(u)\|
$$

and this tends to zero as $h \rightarrow 0^{+}$. So $H$ is continuous.

Lemma 11 ([2, p. 216]). If $F: \mathbb{R} \rightarrow c c(X)$ is continuous, then

$$
\lim _{h \rightarrow 0^{+}} \frac{1}{h} \int_{t}^{t+h} F(u) d u=F(t) \quad \text { for } t \in \mathbb{R} .
$$

Lemma 12. If $F:[0, \infty) \rightarrow c c(X)$ is continuous, then

$$
\int_{0}^{t}\left(\int_{0}^{s} F(u) d u\right) d s=\int_{0}^{t}(t-u) F(u) d u \quad \text { for } t \geq 0 .
$$

Proof. We define

$$
\phi(t):=d\left(\int_{0}^{t}\left(\int_{0}^{s} F(u) d u\right) d s, \int_{0}^{t}(t-u) F(u) d u\right) \quad \text { for } t \geq 0 .
$$

Lemma 10 implies that $\phi$ is continuous. By Lemma 3 and properties of the Hausdorff metric for $h>0$ we have

$$
\begin{aligned}
& \phi(t+h) \\
& =d\left(\int_{0}^{t+h}\left(\int_{0}^{s} F(u) d u\right) d s, \int_{0}^{t+h}(t+h-u) F(u) d u\right) \\
& \leq d\left(\int_{0}^{t}\left(\int_{0}^{t} F(u) d u\right) d s, \int_{0}^{t}(t-u) F(u) d u\right) \\
& +d\left(\int_{t}^{t+h}\left(\int_{0}^{s} F(u) d u\right) d s, \int_{t}^{t+h}(t+h-u) F(u) d u+h \int_{0}^{t} F(u) d u\right) .
\end{aligned}
$$

Thus

$$
\begin{aligned}
& \frac{\phi(t+h)-\phi(t)}{h} \\
& \leq d\left(\frac{1}{h} \int_{t}^{t+h}\left(\int_{0}^{s} F(u) d u\right) d s, \frac{1}{h} \int_{t}^{t+h}(t+h-u) F(u) d u+\int_{0}^{t} F(u) d u\right)
\end{aligned}
$$


for $t \geq 0, h>0$. Since $F$ is continuous there exists $M>0$ such that $\|F(u)\| \leq M$ for $u \in[t, t+1]$. Therefore by Lemma 9 we get

$$
\left\|\frac{1}{h} \int_{t}^{t+h}(t+h-u) F(u) d u\right\| \leq \frac{1}{h} \int_{t}^{t+h}(t+h-u)\|F(u)\| d u \leq \frac{M h}{2}
$$

for $0<h<1$. This implies that

$$
\lim _{h \rightarrow 0^{+}} \frac{1}{h} \int_{t}^{t+h}(t+h-u) F(u) d u=\{0\} .
$$

Using the last equality, (4), Lemmas 10 and 11 we have

$$
\begin{aligned}
& \liminf _{h \rightarrow 0^{+}} \frac{\phi(t+h)-\phi(t)}{h} \\
& \leq \lim _{h \rightarrow 0^{+}} d\left(\frac{1}{h} \int_{t}^{t+h}\left(\int_{0}^{s} F(u) d u\right) d s, \int_{0}^{t} F(u) d u\right) \\
& +\lim _{h \rightarrow 0^{+}}\left\|\frac{1}{h} \int_{t}^{t+h}(t+h-u) F(u) d u\right\| \\
& =d\left(\int_{0}^{t} F(u) d u, \int_{0}^{t} F(u) d u\right)+0=0 .
\end{aligned}
$$

According to a corollary from the Zygmund Lemma in $[5$, p. 174] the function $\phi$ is nonincreasing. Therefore

$$
\phi(t) \leq \phi(0)=0
$$

for $t \geq 0$. This shows that equality (3) holds.

Lemma 13. If $F:[0, \infty) \rightarrow c c(X)$ is continuous, then

$$
\int_{0}^{t}\left(\frac{(t-s)^{n}}{n !} \int_{0}^{s}(s-u) F(u) d u\right) d s=\int_{0}^{t} \frac{(t-u)^{n+2}}{(n+2) !} F(u) d u
$$

for $t \geq 0, n=0,1,2 \ldots$

Proof. For every nonnegative integer $n$ we define

$$
\phi_{n}(t)=d\left(\int_{0}^{t}\left(\frac{(t-s)^{n}}{n !} \int_{0}^{s}(s-u) F(u) d u\right) d s, \int_{0}^{t} \frac{(t-u)^{n+2}}{(n+2) !} F(u) d u\right) .
$$


For $n=0$ we have

$$
\phi_{0}(t)=d\left(\int_{0}^{t}\left(\int_{0}^{s}(s-u) F(u) d u\right) d s, \int_{0}^{t} \frac{(t-u)^{2}}{2 !} F(u) d u\right)
$$

and by Lemma 3 and properties of the Hausdorff metric we have

$$
\begin{aligned}
& \phi_{0}(t+h) \\
& =d\left(\int_{0}^{t}\left(\int_{0}^{s}(s-u) F(u) d u\right) d s+\int_{t}^{t+h}\left(\int_{0}^{s}(s-u) F(u) d u\right) d s,\right. \\
& \int_{0}^{t} \frac{(t-u)^{2}}{2 !} F(u) d u+h \int_{0}^{t}(t-u) F(u) d u \\
& \left.+\frac{h^{2}}{2} \int_{0}^{t} F(u) d u+\int_{t}^{t+h} \frac{(t+h-u)^{2}}{2 !} F(u) d u\right) \\
& \leq \phi_{0}(t)+d\left(\int_{t}^{t+h}\left(\int_{0}^{s}(s-u) F(u) d u\right) d s, h \int_{0}^{t}(t-u) F(u) d u\right. \\
& \left.+\frac{h^{2}}{2} \int_{0}^{t} F(u) d u+\int_{t}^{t+h} \frac{(t+h-u)^{2}}{2 !} F(u) d u\right)
\end{aligned}
$$

for $h>0$. Therefore

$$
\begin{aligned}
& \frac{\phi_{0}(t+h)-\phi_{0}(t)}{h} \\
& \leq d\left(\frac{1}{h} \int_{t}^{t+h}\left(\int_{0}^{s}(s-u) F(u) d u\right) d s, \int_{0}^{t}(t-u) F(u) d u\right) \\
& +\frac{h}{2}\left\|\int_{0}^{t} F(u) d u\right\|+\left\|\frac{1}{h} \int_{t}^{t+h} \frac{(t+h-u)^{2}}{2 !} F(u) d u\right\| .
\end{aligned}
$$

Since

$$
\left\|\frac{1}{h} \int_{t}^{t+h} \frac{(t+h-u)^{2}}{2 !} F(u) d u\right\|
$$




$$
\leq \frac{1}{h} \int_{t}^{t+h} \frac{(t+h-u)^{2}}{2 !} d u \sup _{t \leq u \leq t+h}\|F(u)\|=\frac{h^{2}}{6} \sup _{t \leq u \leq t+h}\|F(u)\|
$$

we have

$$
\lim _{h \rightarrow 0^{+}} \frac{1}{h} \int_{t}^{t+h} \frac{(t+h-u)^{2}}{2 !} F(u) d u=\{0\}
$$

and

$$
\liminf _{h \rightarrow 0^{+}} \frac{\phi_{0}(t+h)-\phi_{0}(t)}{h} \leq 0 .
$$

As we know the function $\phi_{0}$ is continuous nonnegative and $\phi_{0}(0)=0$, whence by the corollary from the Zygmund Lemma $\phi_{0}(t)=0$ for $t \geq 0$. Thus

$$
\int_{0}^{t}\left(\int_{0}^{s}(s-u) F(u) d u\right) d s=\int_{0}^{t} \frac{(t-u)^{2}}{2 !} F(u) d u .
$$

Let $n$ be a nonnegative integer. Suppose that $\phi_{n} \equiv 0$. By Lemma 3 and properties of the Hausdorff metric for $h>0$ we have

$$
\begin{aligned}
& \phi_{n+1}(t+h) \\
& =d\left(\int_{0}^{t+h}\left(\frac{(t+h-s)^{n+1}}{(n+1) !} \int_{0}^{s}(s-u) F(u) d u\right) d s\right. \\
& \left.\int_{0}^{t+h} \frac{(t+h-u)^{n+3}}{(n+3) !} F(u) d u\right) \\
& \leq \phi_{n+1}(t)+d\left(\sum_{i=0}^{n}\left(\begin{array}{c}
n+1 \\
i
\end{array}\right)\left(\int_{0}^{t} \frac{(t-s)^{i} h^{n+1-i}}{(n+1) !} \int_{0}^{s}(s-u) F(u) d u\right) d s\right. \\
& +\int_{t}^{t+h}\left(\frac{(t+h-s)^{n+1}}{(n+1) !} \int_{0}^{t}(s-u) F(u) d u\right) d s, \\
& \left.\sum_{j=0}^{n+2}\left(\begin{array}{c}
n+3 \\
j
\end{array}\right) \int_{0}^{t} \frac{(t-u)^{j} h^{n+3-j}}{(n+3) !} F(u) d u+\int_{t}^{t+h} \frac{(t+h-u)^{n+3}}{(n+3) !} F(u) d u\right) .
\end{aligned}
$$

Therefore

$$
\frac{\phi_{n+1}(t+h)-\phi_{n+1}(t)}{h}
$$




$$
\begin{aligned}
& \leq d\left(\sum_{i=0}^{n}\left(\begin{array}{c}
n+1 \\
i
\end{array}\right)\left(\int_{0}^{t} \frac{(t-s)^{i} h^{n-i}}{(n+1) !} \int_{0}^{s}(s-u) F(u) d u\right) d s\right. \\
& +\frac{1}{h} \int_{t}^{t+h}\left(\frac{(t+h-s)^{n+1}}{(n+1) !} \int_{0}^{s}(s-u) F(u) d u\right) d s, \\
& \sum_{j=0}^{n+2}\left(\begin{array}{c}
n+3 \\
j
\end{array}\right) \int_{0}^{t} \frac{(t-u)^{j} h^{n+2-j}}{(n+3) !} F(u) d u \\
& \left.+\frac{1}{h} \int_{t}^{t+h} \frac{(t+h-u)^{n+3}}{(n+3) !} F(u) d u\right) .
\end{aligned}
$$

Since

$$
\left\|\frac{1}{h} \int_{t}^{t+h}\left(\frac{(t+h-s)^{n+1}}{(n+1) !} \int_{0}^{s}(s-u) F(u) d u\right) d s\right\| \leq M \frac{h^{n+1}(t+h)^{2}}{2(n+2) !}
$$

and

$$
\left\|\frac{1}{h} \int_{t}^{t+h} \frac{(t+h-u)^{n+3}}{(n+3) !} F(u) d u\right\| \leq M \frac{h^{n+3}}{(n+4) !}
$$

for $0<h<1$, where $M=\sup \{\|F(u)\|: t \leq u \leq t+1\}$, we have

$$
\begin{aligned}
& \liminf _{h \rightarrow 0^{+}} \frac{\phi_{n+1}(t+h)-\phi_{n+1}(t)}{h} \\
& \leq d\left(\int_{0}^{t}\left(\frac{(t-s)^{n}}{n !} \int_{0}^{s}(s-u) F(u) d u\right) d s, \int_{0}^{t} \frac{(t-u)^{n+2}}{(n+2) !} F(u) d u\right) \\
& =\phi_{n}(t)=0 .
\end{aligned}
$$

Applying the corollary from the Zygmund Lemma we obtain that $\phi_{n+1} \equiv 0$ and equality (5) holds for every $n \geq 0$ and $t \geq 0$.

It is not difficult to check that the following lemma is true.

Lemma 14. Let $K$ be a convex cone in $X$. If $F: K \rightarrow c c(X)$ is linear continuous, $G:[a, b] \rightarrow c c(K)$ is continuous, then

$$
\int_{a}^{b} F(G(t)) d t=F\left(\int_{a}^{b} G(t) d t\right) .
$$


Let $F, G: K \rightarrow c c(K)$. We can define the multifunctions $F+G$ and $F-G$ on $K$ as follows

$$
(F+G)(x):=F(x)+G(x) \text { for } x \in K
$$

and

$$
(F-G)(x):=F(x)-G(x)
$$

if the Hukuhara differences $F(x)-G(x)$ exist for all $x \in K$.

Lemma 15 ([11, Lemma 2]). For each set $A \subset K$ the inclusion

$$
(F+G)(A) \subset F(A)+G(A)
$$

holds. Moreover, if there exist the Hukuhara difference $F(A)-G(A)$ and the multifunction $F-G$, then

$$
F(A)-G(A) \subset(F-G)(A) .
$$

Let $K$ be a closed convex cone in a real Banach space. Applying Theorem 4 in [14] we define the norm of a linear continuous multifunction $F: K \rightarrow n(K)$, denoted by $\|F\|$, to be the smallest element of the set

$$
\{M \geq 0:\|F(x)\| \leq M\|x\|, x \in K\} .
$$

Theorem 1. Let $X$ be a real Banach space and let $K$ be a closed convex cone in $X$. Assume that $H: K \rightarrow c c(K)$ is a linear continuous multifunction. Then for every $x \in K$ and $t \geq 0$ the series

$$
F_{t}(x)=\sum_{n=0}^{\infty} \frac{t^{2 n}}{(2 n) !} H^{n}(x)
$$

is convergent in the metric space $(c c(K), d)$. Moreover, the multifunctions $F_{t}, t \geq 0$ are linear and

$$
\left(2 F_{t} \circ F_{s}\right)(x) \subset F_{t+s}(x)+F_{t-s}(x)
$$

for $x \in K, t \geq s \geq 0$.

The proof is similar to the proof of Theorem in [9].

It is obvious that for single-valued functions we have the equality instead of the inclusion in the assertion of Theorem 1 (see also Theorem 3.3 in [15]).

Theorem 2. Let $X$ be a real Banach space and let $K$ be a closed convex cone in $X$. If $H: K \rightarrow c c(K)$ is a linear continuous multifunction such that $x \in H(x)$ and $H^{2}(x)=H(x)$ for $x \in K$, then the multifunction given by (8) satisfies the equation

$$
F_{t+s}(x)+F_{t-s}(x)=2 F_{t}\left(F_{s}(x)\right)
$$

for $x \in K, s, t \in \mathbb{R}$. 
Proof. By the assumption $H^{2}(x)=H(x)$ we have

$$
F_{t}(x)=\sum_{n=0}^{\infty} \frac{t^{2 n}}{(2 n) !} H^{n}(x)=x+\sum_{n=1}^{\infty} \frac{t^{2 n}}{(2 n) !} H(x)=x+(\cosh t-1) H(x) .
$$

This equality yields (9).

For example $H:[0, \infty) \rightarrow c c([0, \infty))$ defined by $H(x)=[0, x]$ or $H:[0, \infty)^{2} \rightarrow c c\left([0, \infty)^{2}\right)$ defined by $H((x, y))=[0, x] \times[0, y]$ satisfy the assumptions of Theorem 2 .

Definition. Let $(K,+)$ be a semigroup. A one-parameter family $\left\{F_{t}: t \in \mathbb{R}\right\}$ of multifunctions $F_{t}: K \rightarrow n(K)$ is said to be a cosine family if

$$
F_{0}(x)=\{x\} \quad \text { for } x \in K
$$

and

$$
F_{t+s}+F_{t-s}=2 F_{t} \circ F_{s}
$$

on $K$ for $s, t \in \mathbb{R}$.

Let $X$ be a real normed space. A cosine family $\left\{F_{t}: t \in \mathbb{R}\right\}$ is regular if

$$
\lim _{t \rightarrow 0} d\left(F_{t}(x),\{x\}\right)=0 .
$$

Cosine families of single-valued functions was considered by many authors. These families are relate to second order differential equations (for example see [3], [16]). J. Kisyński proved that solution of some second order differential problem is a cosine family (see Lemma 1.3.3 in [3]).

Lemma 16 ([11, Lemma 8]). Let $X$ be a Banach space and let $K$ be a closed convex cone in $X$ such that int $K \neq \emptyset$. Assume that $\left\{F_{t}: t \in \mathbb{R}\right\}$ is a regular cosine family of continuous additive set-valued functions $F_{t}$ : $K \rightarrow c c(K)$ and $x \in F_{t}(x)$ for all $x \in K$ and $t \in \mathbb{R}$. Then there exist the Hukuhara differences $F_{t}(x)-F_{s}(x)$ for all $0 \leq s \leq t$ and $x \in K$.

Theorem 3. Let $X$ be a real Banach space and let $K$ be a closed convex cone in $X$ such that int $K \neq \emptyset$. Assume that $\left\{F_{t}: t \in \mathbb{R}\right\}$ is a regular cosine family of continuous linear multifunctions $F_{t}: K \rightarrow c c(X)$ and $x \in F_{t}(x)$ for all $x \in K$ and $t \in \mathbb{R}$. Then there exists a continuous linear multifunction $H: K \rightarrow c c(K)$ such that

$$
F_{t}(x) \subset \sum_{n=0}^{\infty} \frac{t^{2 n}}{(2 n) !} H^{n}(x)
$$

for $x \in K$ and $t \in \mathbb{R}$. 
Proof. Let $x \in K$. Consider the multifunction $t \mapsto F_{t}(x)$ for $t \in \mathbb{R}$. We first show some properties of this multifunction. Putting $t=0$ in (10) we have $F_{s}(x)+F_{-s}(x)=2 F_{0}\left(F_{s}(x)\right)=2 F_{s}(x)=F_{s}(x)+F_{s}(x)$. By Rådström Lemma we obtain $F_{s}(x)=F_{-s}(x)$. Thus the multifunction $t \mapsto F_{t}(x)$ is even. Hence and by (10) we have $F_{t} \circ F_{s}=F_{s} \circ F_{t}$. According to Theorem 2 in [13] the multifunction $t \mapsto F_{t}(x)$ is continuous on $[0,+\infty)$. As $t \mapsto F_{t}(x)$ is even it is continuous on $\mathbb{R}$. By Lemma 8 it is integrable on each interval $[a, b] \subset \mathbb{R}$. Setting $t=(v+u) / 2, s=(v-u) / 2, u, v \in \mathbb{R}$ in (10) we get

$$
F_{v}(x)+F_{u}(x)=2 F_{(v+u) / 2}\left(F_{(v-u) / 2}(x)\right) .
$$

Since $x \in F_{t}(x)$ we have

$$
F_{(v+u) / 2}(x) \subset \frac{F_{v}(x)+F_{u}(x)}{2} .
$$

Hence, by the continuity and Theorem 1 in [7] the multifunction $t \mapsto F_{t}(x)$ is concave. We observe that $t \mapsto F_{t}(x)$ is increasing in $[0,+\infty)$ and decreasing in $(-\infty, 0]$. Indeed, for $0 \leq s \leq t$ there exists $\lambda \in[0,1]$ such that $s=$ $(1-\lambda) 0+\lambda t$. Hence $F_{s}(x) \subset(1-\lambda) F_{0}(x)+\lambda F_{t}(x)=(1-\lambda) x+\lambda F_{t}(x) \subset$ $(1-\lambda) F_{t}(x)+\lambda F_{t}(x)=F_{t}(x)$. For $t \leq s \leq 0$ we have $F_{s}(x)=F_{-s}(x) \subset$ $F_{-t}(x)=F_{t}(x)$.

We next define some multifunctions. We use them later in the construction of the multifunction $H$ and in proving of the inclusion in the assertion. To define these multifuctions we need to show the existence of some limits. Take arbitrary $\alpha$ and $t$ such that $0<\alpha<t$. Integrating over $s \in[0, \alpha]$ formula (10) we obtain

$$
\int_{0}^{\alpha} F_{t+s}(x) d s+\int_{0}^{\alpha} F_{t-s}(x) d s=\int_{0}^{\alpha} 2 F_{t}\left(F_{s}(x)\right) d s .
$$

Hence, by Lemmas 4, 6 and 14 we have

$$
\int_{t}^{\alpha+t} F_{w}(x) d w+\int_{t-\alpha}^{t} F_{w}(x) d w=2 F_{t}\left(\int_{0}^{\alpha} F_{s}(x) d s\right) .
$$

The last equality and Lemma 2 lead to

$$
\int_{t-\alpha}^{t+\alpha} F_{w}(x) d w=2 F_{t}\left(\int_{0}^{\alpha} F_{s}(x) d s\right) .
$$

Replacing in (11) $t$ by $t+u$, where $u \in(0, \alpha)$, we get

$$
\int_{t-\alpha+u}^{t+\alpha+u} F_{w}(x) d w=2 F_{t+u}\left(\int_{0}^{\alpha} F_{s}(x) d s\right) .
$$


Adding both the sides (11) and (12) we have

$$
\begin{aligned}
& \int_{t-\alpha+u}^{t+\alpha+u} F_{w}(x) d w+2 F_{t}\left(\int_{0}^{\alpha} F_{s}(x) d s\right) \\
& =\int_{t-\alpha}^{t+\alpha} F_{w}(x) d w+2 F_{t+u}\left(\int_{0}^{\alpha} F_{s}(x) d s\right) .
\end{aligned}
$$

Then we get by Lemma 3 substracting the term $\int_{t-\alpha+u}^{t+\alpha} F_{w}(x) d w$ from both the sides of the last equality

$$
\begin{aligned}
& \int_{t+\alpha}^{t+\alpha+u} F_{w}(x) d w+2 F_{t}\left(\int_{0}^{\alpha} F_{s}(x) d s\right) \\
& =\int_{t-\alpha}^{t-\alpha+u} F_{w}(x) d w+2 F_{t+u}\left(\int_{0}^{\alpha} F_{s}(x) d s\right) .
\end{aligned}
$$

By Lemma 7 the Hukuhara difference

$$
\int_{t+\alpha}^{t+\alpha+u} F_{w}(x) d w-\int_{t-\alpha}^{t-\alpha+u} F_{w}(x) d w
$$

exists, which together with (13) shows that the Hukuhara difference

$$
2 F_{t+u}\left(\int_{0}^{\alpha} F_{s}(x) d s\right)-2 F_{t}\left(\int_{0}^{\alpha} F_{s}(x) d s\right)
$$

also exists and

$$
\begin{aligned}
& \frac{2 F_{t+u}\left(\int_{0}^{\alpha} F_{s}(x) d s\right)-2 F_{t}\left(\int_{0}^{\alpha} F_{s}(x) d s\right)}{u} \\
& =\frac{1}{u} \int_{t+\alpha}^{t+\alpha+u} F_{w}(x) d w-\frac{1}{u} \int_{t-\alpha}^{t-\alpha+u} F_{w}(x) d w .
\end{aligned}
$$

In virtue of Lemmas 1 and 11 there exists

$$
\lim _{u \rightarrow 0^{+}} \frac{2 F_{t+u}\left(\int_{0}^{\alpha} F_{s}(x) d s\right)-2 F_{t}\left(\int_{0}^{\alpha} F_{s}(x) d s\right)}{u}=F_{t+\alpha}(x)-F_{t-\alpha}(x) .
$$


We define

$$
\begin{aligned}
G_{\alpha, t}(x) & :=\frac{F_{t+\alpha}(x)-F_{t-\alpha}(x)}{2} \\
& =\lim _{u \rightarrow 0^{+}} \frac{F_{t+u}\left(\int_{0}^{\alpha} F_{s}(x) d s\right)-F_{t}\left(\int_{0}^{\alpha} F_{s}(x) d s\right)}{u} .
\end{aligned}
$$

We observe that

$$
G_{\alpha, t}(x)=\frac{F_{t+\alpha}(x)-F_{t-\alpha}(x)}{2}=\frac{F_{\alpha+t}(x)-F_{\alpha-t}(x)}{2}=G_{t, \alpha}(x) .
$$

Since the multifunction $s \mapsto F_{s}(x)$ is concave and there exist the Hukuhara differences $F_{t}(x)-F_{s}(x)$ for $t \geq s \geq 0$, so by Theorem 3.2 in [10] there exist

$$
\begin{aligned}
G_{t}^{+}(x) & :=\lim _{\alpha \rightarrow 0^{+}} \frac{F_{t+\alpha}(x)-F_{t}(x)}{\alpha}, \\
G_{t}^{-}(x) & :=\lim _{\alpha \rightarrow 0^{+}} \frac{F_{t}(x)-F_{t-\alpha}(x)}{\alpha}
\end{aligned}
$$

and $G_{t}^{-}(x) \subset G_{t}^{+}(x)$ for $t>0$. Consequently there exists

$$
G_{t}(x):=\lim _{\alpha \rightarrow 0^{+}} \frac{F_{t+\alpha}(x)-F_{t-\alpha}(x)}{\alpha}=G_{t}^{+}(x)+G_{t}^{-}(x) \text { for } t>0 .
$$

It follows from (10) that

$$
\frac{F_{2 t}(x)-x}{2 t}=F_{t}\left(\frac{F_{t}(x)-x}{t}\right)+\frac{F_{t}(x)-x}{t} .
$$

Letting $t \rightarrow 0^{+}$we get

$$
\lim _{t \rightarrow 0^{+}} F_{t}\left(\frac{F_{t}(x)-x}{t}\right)=\{0\}
$$

and since

$$
0 \in \frac{F_{t}(x)-x}{t} \subset F_{t}\left(\frac{F_{t}(x)-x}{t}\right)
$$

we have

$$
G_{0}^{+}(x):=\lim _{t \rightarrow 0^{+}} \frac{F_{t}(x)-x}{t}=\{0\} .
$$

Our next claim is that the multifunction $t \mapsto G_{t}^{+}(x)$ is concave. Replacing in (10) $t$ by $t+u, u>0$ and substract $F_{t+s}(x)+F_{t-s}(x)$ from both the sides of this equality we get

$$
F_{t+s+u}(x)-F_{t+s}(x)+F_{t-s+u}(x)-F_{t-s}(x)=2 F_{t+u}\left(F_{s}(x)\right)-2 F_{t}\left(F_{s}(x)\right) .
$$


Dividing the last equality by $u$ we get

$$
\frac{F_{t+s+u}(x)-F_{t+s}(x)}{u}+\frac{F_{t-s+u}(x)-F_{t-s}(x)}{u}=2 F_{s}\left(\frac{F_{t+u}(x)-F_{t}(x)}{u}\right)
$$

and letting $u \rightarrow 0^{+}$we obtain

$$
G_{t+s}^{+}(x)+G_{t-s}^{+}(x)=2 F_{s}\left(G_{t}^{+}(x)\right) .
$$

Setting $t=(v+u) / 2, s=(v-u) / 2, u, v \in \mathbb{R}$ in (15) we have

$$
G_{v}^{+}(x)+G_{u}^{+}(x)=2 F_{(v-u) / 2}\left(G_{(v+u) / 2}^{+}(x)\right) .
$$

By assumption $x \in F_{t}(x)$ we get

$$
G_{(v+u) / 2}^{+}(x) \subset \frac{G_{v}^{+}(x)+G_{u}^{+}(x)}{2} .
$$

Fix an interval $[a, b] \subset[0, \infty)$ and let $t \in[a, b]$. Since $G_{t}^{+}(x) \subset$ $F_{t+1}(x)-F_{t}(x)$ we have

$$
G_{t}^{+}(x)+x \subset G_{t}^{+}(x)+F_{t}(x) \subset F_{t+1}(x) \subset F_{b+1}(x) .
$$

Therefore the multifunction $t \mapsto G_{t}^{+}(x)$ is bounded on $[a, b]$. By Theorem 4.4 in [6] the multifunction $t \mapsto G_{t}(x)$ is continuous in $(0, \infty)$ and concave by Theorem 4.1 in $[6]$. Let $\lambda \in(0,1)$. Then $G_{\lambda t}^{+}(x) \subset \lambda G_{t}^{+}(x)$ and

$$
\frac{G_{\lambda t}^{+}(x)}{\lambda t} \subset \frac{G_{t}^{+}(x)}{t}
$$

it follows that there exists

$$
\lim _{t \rightarrow 0^{+}} \frac{G_{t}^{+}(x)}{t}=: H(x)
$$

Since $x \mapsto F_{t}(x)$ for every $t \in \mathbb{R}$ are linear continuous we see $H$ is linear and $\|H(x)\| \leq\left\|G_{1}(x)\right\| \leq\left\|G_{1}\right\|\|x\|$, hence $H$ is continuous, too.

By uniform convergence of $\lim _{u \rightarrow 0^{+}}\left(F_{\alpha+u}(x)-F_{\alpha}(x)\right) / u$ on each compact subset of $K$ (see Theorem 1 in [8]), Lemma 15 and equality (14) we 
get

$$
\begin{aligned}
& 2 G_{t}^{-}(x) \\
& \subset G_{t}(x)=\lim _{\alpha \rightarrow 0^{+}} \frac{2}{\alpha} G_{\alpha, t}(x)=\lim _{\alpha \rightarrow 0^{+}} \frac{2}{\alpha} G_{t, \alpha}(x) \\
& =\lim _{\alpha \rightarrow 0^{+}} \frac{2}{\alpha} \lim _{u \rightarrow 0^{+}} \frac{F_{\alpha+u}\left(\int_{0}^{t} F_{s}(x) d s\right)-F_{\alpha}\left(\int_{0}^{t} F_{s}(x) d s\right)}{u} \\
& \subset \lim _{\alpha \rightarrow 0^{+}} \frac{2}{\alpha} \lim _{u \rightarrow 0^{+}} \frac{\left(F_{\alpha+u}-F_{\alpha}\right)\left(\int_{0}^{t} F_{s}(x) d s\right)}{u} \\
& =\lim _{\alpha \rightarrow 0^{+}} \frac{2}{\alpha} G_{\alpha}^{+}\left(\int_{0}^{t} F_{s}(x) d s\right)=2 H\left(\int_{0}^{t} F_{s}(x) d s\right) .
\end{aligned}
$$

According to Theorem 4.3 in [10] we have

$$
F_{t}(x)=x+\int_{0}^{t} G_{s}^{-}(x) d s .
$$

Hence, by (16), Lemmas 5, 12 and 14 we obtain

$$
\begin{aligned}
F_{t}(x) \subset x+\int_{0}^{t} H\left(\int_{0}^{s} F_{u}(x) d u\right) d s \\
=x+H\left(\int_{0}^{t}\left(\int_{0}^{s} F_{u}(x) d u\right) d s\right)=x+H\left(\int_{0}^{t}(t-u) F_{u}(x) d u\right) .
\end{aligned}
$$

Using Lemma 13 instead of Lemma 12 we get succesively

$$
\begin{aligned}
F_{t}(x) & \subset x+H\left[\int_{0}^{t}(t-u)\left(x+H\left(\int_{0}^{u}(u-s) F_{s}(x) d s\right)\right) d u\right] \\
& =x+H\left[\int_{0}^{t}(t-u) x d u+\int_{0}^{t}(t-u) H\left(\int_{0}^{u}(u-s) F_{s}(x) d s\right) d u\right] \\
& \left.=x+H\left(\frac{1}{2} t^{2} x\right)+H^{2}\left[\int_{0}^{t}(t-u) \int_{0}^{u}(u-s) F_{s}(x) d s\right) d u\right]
\end{aligned}
$$




$$
=x+\frac{t^{2}}{2} H(x)+H^{2}\left(\int_{0}^{t} \frac{(t-s)^{3}}{3 !} F_{s}(x) d s\right) .
$$

Repeating the same steps we have

$$
\begin{aligned}
F_{t}(x) & \subset x+\frac{t^{2}}{2 !} H(x)+\ldots+\frac{t^{2 n}}{(2 n) !} H^{n}(x) \\
& +H^{n+1}\left(\int_{0}^{t} \frac{(t-s)^{2 n+1}}{(2 n+1) !} F_{s}(x) d s\right) .
\end{aligned}
$$

It remains to prove that

$$
H^{n+1}\left(\int_{0}^{t} \frac{(t-s)^{2 n+1}}{(2 n+1) !} F_{s}(x) d s\right) \rightarrow\{0\} .
$$

For $s \in[0, t], F_{s}(x) \subset F_{t}(x)$, so $\left\|F_{s}(x)\right\| \leq\left\|F_{t}(x)\right\|$. Let $m=\left\|F_{t}(x)\right\|$. By Lemma 9 we have

$$
\begin{aligned}
& \left\|H^{n+1}\left(\int_{0}^{t} \frac{(t-s)^{2 n+1}}{(2 n+1) !} F_{s}(x) d s\right)\right\| \\
& \leq\|H\|^{n+1}\left\|\int_{0}^{t} \frac{(t-s)^{2 n+1}}{(2 n+1) !} F_{s}(x) d s\right\| \\
& \leq\|H\|^{n+1} \int_{0}^{t} \frac{(t-s)^{2 n+1}}{(2 n+1) !}\left\|F_{s}(x)\right\| d s \\
& \leq\|H\|^{n+1} m \int_{0}^{t} \frac{(t-s)^{2 n+1}}{(2 n+1) !} d s \\
& \leq\|H\|^{n+1} m \frac{t^{2 n+2}}{(n+1) !}=\frac{\left(\|H\| t^{2}\right)^{n+1}}{(n+1) !} m .
\end{aligned}
$$

Since

$$
\frac{\left(\|H\| t^{2}\right)^{n+1}}{(n+1) !} m \rightarrow 0
$$

we have

$$
H^{n+1}\left(\int_{0}^{t} \frac{(t-s)^{2 n+1}}{(2 n+1) !} F_{s}(x) d s\right) \rightarrow\{0\} .
$$


Therefore

$$
F_{t}(x) \subset \sum_{n=0}^{\infty} \frac{t^{2 n}}{(2 n) !} H^{n}(x) \quad \text { for } t \in \mathbb{R}, x \in K .
$$

S. Kurepa in [4] proved that every single-valued function of the cosine family is in the form of the series. Theorem 3 is not generalization of Theorems for single-valued case.

Example 1. A family $\left\{F_{t}: t \in \mathbb{R}\right\}$ of multifunctions $F_{t}:[0, \infty) \rightarrow$ $c c([0, \infty))$ such that $F_{t}(x)=x[1, \cosh t]$ is a regular cosine family. Our wanted multifunction is $H(x)=[0, x]$ and

$$
F_{t}(x)=\sum_{n=0}^{\infty} \frac{t^{2 n}}{(2 n) !} H^{n}(x)=x+\sum_{n=1}^{\infty} \frac{t^{2 n}}{(2 n) !}[0, x] .
$$

Example 2. A family $\left\{F_{t}: t \in \mathbb{R}\right\}$ of multifunctions $F_{t}:[0, \infty)^{2} \rightarrow$ $c c\left([0, \infty)^{2}\right)$ defined by $F_{t}((x, y))=[x, x \cosh t] \times[y, y \cosh t]$ is a regular cosine family. Our wanted multifunction is $H((x, y))=[0, x] \times[0, y]$ and

$$
F_{t}((x, y))=\sum_{n=0}^{\infty} \frac{t^{2 n}}{(2 n) !} H^{n}((x, y))=(x, y)+\sum_{n=1}^{\infty} \frac{t^{2 n}}{(2 n) !}[0, x] \times[0, y] .
$$

\section{References}

[1] Castaing, Ch., Valadier, M., Convex Analysis and Measurable Multifunctions, Lecture Notes in Math. 580, Springer, New York, 1977.

[2] Hukuhara, M., Intégration des application mesurables dont la valeur est un compact convexe, Funkcial. Ekvac. 10 (1967), 205-223.

[3] Kisyński, J., On cosine operator functions and one-parameter groups of operators, Studia Math. 44 (1972), 93-105.

[4] Kurepa, S., A cosine function equation in Banach algebras, Acta Sci. Math. (Szeged) 23 (1962), 255-267.

[5] Eojasiewicz, S., An Introduction to the Theory of Real Functions, A WilleyInterscience Publication, John Willey \& Sons, Ltd., Chichester, 1988.

[6] Nikodem, K., $K$-convex and $K$-concave set-valued function, Zeszyty Nauk. Politech. Łódz. Mat. 559, Rozprawy Nauk. 144, Łódź, 1989.

[7] Nikodem, K., On concave and midpoint concave set-valued function, Glas. Mat. Ser. III 22 (1987), 69-76.

[8] Olko, J., Concave interetion semigroups of linear set-valued functions, Ann. Polon. Math. 71 (1999), 31-38.

[9] Plewnia, J., On a family of a set-valued functions, Publ. Math. Debrecen 46 (1995), 149-159. 
[10] Piszczek, M., Integral representations of convex and concave set-valued functions, Demonstratio Math. 35 (2002), 727-742.

[11] Piszczek, M., Second Hukuhara derivative and cosine family of linear set-valued functions, Ann. Acad. Paedagogicae Cracoviensis, Studia Math. 5 (2006), 87-98.

[12] Rådström, H., An embeldding theorem for space of convex sets, Proc. Amer. Math. Soc. 3 (1952), 165-169.

[13] Smajdor, A., On regular multivalued cosine families, Ann. Math. Sil. 13 (1999), 271280.

[14] Smajdor, W., Superadditive set-valued functions and Banach-Steinhaus theorem, Rad. Mat. 3 (1987), 203-214.

[15] Sova, M., Cosine operator functions, Rozprawy Mat. 49 (1966), 1-47.

[16] Travis, C. C., Webb, G. F., Cosine families and abstract nonlinear second order differential equations, Acta Math. Hungar. 32 (1978), 75-95.

\author{
Magdalena PisZCZeK \\ Institute of Mathematics \\ PEDAGOGICAL University \\ PODCHORA̧ŻYCH 2 \\ 30-084 Cracow, Poland \\ E-MAIL: MAGDAP@AP.KRAKOW.PL
}

